\title{
On Double Integrals Involving Generalized H-Function of Two Variables
}

\author{
Mehphooj Beg ${ }^{1} \&$ Dr. S. S. Shrivastava ${ }^{2}$ \\ ${ }^{I}$ VITS, Satna (M. P.) \\ ${ }^{2}$ Institute for Excellence in Higher Education Bhopal (M. P.)
}

Abstract: The aim of this paper is to derive a double integrals involving generalized H-function of two variables.

\section{Introduction}

The generalized H-function of two variables is given by Shrivastava, H. S. P. [4] and defined as follows:

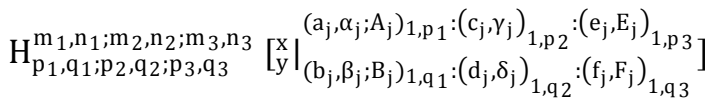

$$
\begin{aligned}
& =\frac{-1}{4 \pi^{2}} \int_{L_{1}} \int_{L_{2}} \phi_{1}(\xi, \eta) \theta_{2}(\xi) \theta_{3}(\eta) x^{\xi} y^{\eta} d \xi d \eta
\end{aligned}
$$

where

$$
\begin{aligned}
& \phi_{1}(\xi, \eta)=\frac{\prod_{j=1}^{n_{1}} \Gamma\left(1-a_{j}+\alpha_{j} \xi+A_{j} \eta\right) \prod_{j=1}^{m} \Gamma\left(b_{j}-\beta_{j} \xi-B_{j} \eta\right)}{\prod_{j=n_{1}+1}^{p_{1}} \Gamma\left(a_{j}-\alpha_{j} \xi-A_{j} \eta\right) \prod_{j=1}^{q_{1}} \Gamma\left(1-b_{j}+\beta_{j} \xi+B_{j} \eta\right)^{\prime}}, \\
& \theta_{2}(\xi)=\frac{\prod_{j=1}^{m_{2}} \Gamma\left(d_{j}-\delta_{j} \xi\right) \prod_{j=1}^{n_{2}} \Gamma\left(1-c_{j}+\gamma_{j} \xi\right)}{\prod_{j=m_{2}+1}^{q} \Gamma\left(1-d_{j}+\delta_{j} \xi\right) \prod_{j=n_{2}+1}^{q_{2}} \Gamma\left(c_{j}-\gamma_{j} \xi\right)} \\
& \theta_{2}(\xi)=\frac{\prod_{j=1}^{m_{3}} \Gamma\left(f_{j}-F_{j} \eta\right) \prod_{j=1}^{n_{3}} \Gamma\left(1-e_{j}+E_{j} \eta\right)}{\prod_{j=m_{3}+1}^{q_{3}} \Gamma\left(1-f_{j}+F_{j} \eta\right) \prod_{j=n_{3}+1}^{p_{3}} \Gamma\left(e_{j}-E_{j} \eta\right)}
\end{aligned}
$$

$\mathrm{x}$ and $\mathrm{y}$ are not equal to zero, and an empty product is interpreted as unity $\mathrm{p}_{\mathrm{i}}, \mathrm{q}_{\mathrm{i}}, \mathrm{n}_{\mathrm{i}}$ and $\mathrm{m}_{\mathrm{j}}$ are non negative integers such that $p_{i} \geq n_{i} \geq 0, q_{i} \geq 0, q_{j} \geq m_{j} \geq 0,(i=1,2,3 ; j=2,3)$. Also, all the A's, $\alpha$ 's, B's, $\beta$ 's, $\gamma$ 's, $\delta$ 's, E's, and F's are assumed to the positive quantities for standardization purpose.

The contour $\mathrm{L}_{1}$ is in the $\xi$-plane and runs from - iळ to $+\mathrm{i} \infty$, with loops, if necessary, to ensure that the poles of $\Gamma\left(\mathrm{d}_{\mathrm{j}}-\delta_{\mathrm{j}} \xi\right)\left(\mathrm{j}=1, \ldots, \mathrm{m}_{2}\right)$ lie to the right, and the poles of $\Gamma\left(1-\mathrm{c}_{\mathrm{j}}+\gamma_{j} \xi\right)\left(\mathrm{j}=1, \ldots, \mathrm{n}_{2}\right), \Gamma\left(1-\mathrm{a}_{\mathrm{j}}+\alpha_{j} \xi+\mathrm{A}_{\mathrm{j}} \eta\right)(\mathrm{j}=1$, $\left.\ldots, \mathrm{n}_{1}\right)$ to the left of the contour.

The contour $L_{2}$ is in the $\eta$-plane and runs from $-i \infty$ to $+i \infty$, with loops, if necessary, to ensure that the poles of $\Gamma\left(f_{j}-F_{j} \eta\right)\left(j=1, \ldots, m_{3}\right)$ lie to the right, and the poles of $\Gamma\left(1-e_{j}+E_{j} \eta\right)\left(j=1, \ldots, n_{3}\right), \Gamma\left(1-a_{j}+\alpha_{j} \xi+A_{j} \eta\right)(j=$ $\left.1, \ldots, \mathrm{n}_{1}\right)$ to the left of the contour.

The generalized $\mathrm{H}$-function of two variables given by (1) is convergent if

$$
\begin{aligned}
& U=\sum_{j=1}^{n_{1}} \alpha_{j}+\sum_{j=1}^{m_{1}} \beta_{j}+\sum_{j=1}^{n_{2}} \gamma_{j}+\sum_{j=1}^{m_{2}} \delta_{j} \\
& -\sum_{j=n_{1}+1}^{p_{1}} \alpha_{j}-\sum_{j=m_{1}+1}^{q_{1}} \beta_{j}-\sum_{j=n_{2}+1}^{p_{2}} \gamma_{j}-\sum_{j=m_{2}+1}^{q_{2}} \delta_{j} ; \\
& V=\sum_{j=1}^{n_{1}} A_{j}+\sum_{j=1}^{m_{1}} B_{j}+\sum_{j=1}^{n_{3}} E_{j}+\sum_{j=1}^{m_{3}} F_{j} \\
& \quad-\sum_{j=n_{1}+1}^{p_{1}} A_{j}-\sum_{j=m_{1}+1}^{q_{1}} B_{j}-\sum_{j=n_{3}+1}^{p_{3}} E_{j}-\sum_{j=m_{3}+1}^{q_{3}} F_{j},
\end{aligned}
$$

where $|\arg \mathrm{x}|<1 / 2 \mathrm{U} \pi,|\arg \mathrm{y}|<1 / 2 \mathrm{~V} \pi$. 


\section{Result Required}

The following result are required in our present investigation:

From Shrivastava [3, p.1, Eq. (1)]:

$$
\left.S_{n}^{m}[x]=\sum_{u=0}^{[n / m}\right] \frac{(-n) m_{u}}{u !} X^{u} A_{n, u},(n=0,1,2, \ldots),
$$

where $m$ is arbitrary positive integer, and the coefficients $A_{n, u}(n, u \geq 0)$ are arbitrary constants, real or complex.

\section{Main Result}

In this paper we will establish the following integral:

$$
\begin{aligned}
& \int_{0}^{1} \int_{0}^{1} x^{\lambda-1}(1-x)^{\mu-1} y^{\rho-1}(1-y)^{a-2 \rho}(1+t y)^{\rho-a-1}
\end{aligned}
$$

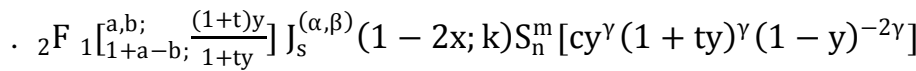

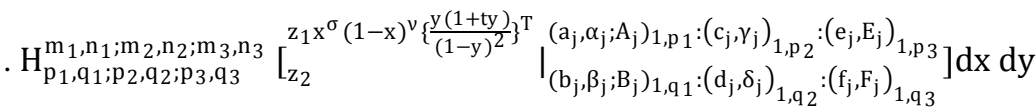

$$
\begin{aligned}
& =\frac{2^{\mathrm{a}}\{4(1+\mathrm{t})\}^{-\rho} \Gamma\left(1+\frac{\mathrm{a}}{2}\right) \Gamma(1+\mathrm{a}-\mathrm{b})(\mathrm{a}+1)_{\mathrm{ks}}}{\sqrt{\pi} \Gamma(1+\mathrm{a}) \Gamma\left(1+\frac{\mathrm{a}}{2}-\mathrm{b}\right) \mathrm{s} !} \sum_{\mathrm{u}=0}^{[\mathrm{n} / \mathrm{m}]} \sum_{\mathrm{j}=0}^{\mathrm{s}} \frac{(-\mathrm{n})_{\mathrm{m}}}{\mathrm{u} !}
\end{aligned}
$$

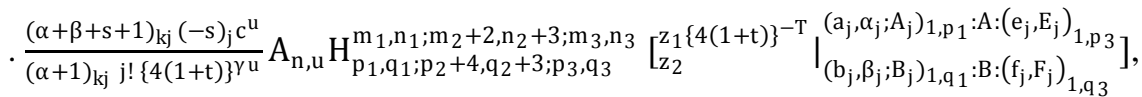

where

$$
\begin{aligned}
& A=(1-\mu, v),(1-\lambda-k j, \sigma),(1-\rho-\gamma u, T),\left(c_{j}, \gamma_{j}\right)_{1, p_{2}},(1+a-b-\rho-\gamma u, T) \\
& B=\left(\frac{1}{2}+\frac{a}{2}-\rho-\gamma u, T\right),\left(1+\frac{a}{2}-b-\rho-\gamma u, T\right),\left(d_{j}, \delta_{j}\right)_{1, q_{2}},(1-\lambda-\mu-k j, \sigma+v) .
\end{aligned}
$$

The integral (5) is valid if the following sets of (sufficient) conditions are satisfied:

(i) $m$ is arbitrary positive integer, and the coefficient $A_{n, u}(n, u \geq 0)$ are arbitrary constants, real or complex.

(ii) $\left|\arg \mathrm{z}_{1}\right|<1 / 2 \mathrm{U} \pi,\left|\arg \mathrm{z}_{2}\right|<1 / 2 \mathrm{~V} \pi$, where $\mathrm{U}$ and $\mathrm{V}$ are given in (2) and (3) respectively.

(iii) $\operatorname{Re}(1+\mathrm{a}-\mathrm{b})>0, \operatorname{Re}(\alpha)>-1, \operatorname{Re}(\beta)>-1, t>-1$,

$$
\begin{aligned}
& \gamma \geq 0, \operatorname{Re}(\lambda+\sigma \xi)>0, \operatorname{Re}(\rho+v \xi)>0, \\
& \operatorname{Re}(\rho+\gamma \mathrm{u}+\mathrm{T} \xi)>0, \operatorname{Re}(1+\mathrm{a}-2 \rho-2 \gamma \mathrm{u}-2 \mathrm{~T} \xi)>0 .
\end{aligned}
$$

(iv) $\operatorname{Re}(1-2 b)>0$.

\section{Proof:}

To establish (5), we use the series representation of $S_{n}^{m}[x]$ as given by (4) and for the generalized Hfunction of two variables we use Mellin-Barnes types of contour integral as given in (1), on the left-hand side of (5), change the order of integration and summation (which is justified under the conditions given with (5)), we then obtain

Left-hand side of $(5)=\sum_{\mathrm{u}=0}^{[\mathrm{n} / \mathrm{m}]} \frac{(-\mathrm{n}) \mathrm{m}_{\mathrm{u}}}{\mathrm{u} !} \mathrm{c}^{\mathrm{u}} A_{\mathrm{n}, \mathrm{u}} \frac{(-1)}{4 \pi^{2}} \int_{\mathrm{L}_{1}} \int_{\mathrm{L}_{2}} \phi_{1}(\xi, \eta) \theta_{2}(\xi) \theta_{3}(\eta)$

$$
\begin{aligned}
& \int_{0}^{1} x^{\lambda+\sigma \xi-1}(1-x)^{\mu+v \xi-1} J_{s}^{(\alpha, \beta)}(1-2 x ; k) d x \\
& \cdot \int_{0}^{1} y^{\rho+\gamma u+T \xi-1}(1-y)^{a-2 T \xi-2 \gamma u-2 \rho}(1+t y)^{\rho-a+\gamma u+T-1} \\
& \left.\cdot{ }_{2} F_{1}\left[{ }_{1+a+b}^{a, b ;} ; \frac{(1+t) y}{1+t y}\right] d y\right] x^{\xi} y^{\eta} d \xi d \eta
\end{aligned}
$$


Now using known results [1, p.118, Eq.(3.3.1)] and [2, p.254, Eq.(2.1)], and interpreting the resulting contour integral as the generalized $\mathrm{H}$-function of two variables, we once get the right-hand side of (5).

\section{Special Cases}

On specializing the parameters in (5), we get following integral in terms of $\mathrm{H}$-function:

$$
\begin{aligned}
& \int_{0}^{1} \int_{0}^{1} x^{\lambda-1}(1-x)^{\mu-1} y^{\rho-1}(1-y)^{a-2 \rho}(1+t y)^{\rho-a-1} \\
& \cdot{ }_{2} \mathrm{~F}_{1}\left[\left[_{1+\mathrm{a}-\mathrm{b} ;}^{\mathrm{a}, \mathrm{b} ;} ; \frac{(1+\mathrm{t}) \mathrm{y}}{1+\mathrm{ty}}\right] \mathrm{J}_{\mathrm{s}}^{(\alpha, \beta)}(1-2 \mathrm{x} ; \mathrm{k}) \mathrm{S}_{\mathrm{n}}^{\mathrm{m}}\left[\mathrm{cy}{ }^{\gamma}(1+\mathrm{ty})^{\gamma}(1-\mathrm{y})^{-2 \gamma}\right]\right. \\
& \cdot H_{P, Q}^{M, N}\left[\left.z^{\sigma}(1-x)^{v}\left\{\frac{y(1+t y)}{(1-y)^{2}}\right\}^{T}\right|_{\left(b_{j}, \beta_{j}\right)_{1, Q}} ^{\left(a_{j}, \alpha_{1}\right)_{1, P}}\right] d x d y \\
& =\frac{2^{\mathrm{a}}\{4(1+\mathrm{t})\}^{-\rho} \Gamma\left(1+\frac{\mathrm{a}}{2}\right) \Gamma(1+\mathrm{a}-\mathrm{b})(\mathrm{a}+1)_{\mathrm{ks}}}{\sqrt{\pi} \Gamma(1+\mathrm{a}) \Gamma\left(1+\frac{\mathrm{a}}{2}-\mathrm{b}\right) \mathrm{s} !} \sum_{\mathrm{u}=0}^{[\mathrm{n} / \mathrm{m}]} \sum_{\mathrm{j}=0}^{\mathrm{s}} \frac{(-\mathrm{n})_{\mathrm{m}_{\mathrm{u}}}}{\mathrm{u} !} \\
& \cdot \frac{(\alpha+\beta+s+1)_{\mathrm{kj}}(-\mathrm{s})_{\mathrm{j}} \mathrm{c}^{\mathrm{u}}}{(\alpha+1)_{\mathrm{kj}} \mathrm{j} !\{4(1+\mathrm{t})\}^{\gamma \mathrm{u}}} \mathrm{A}_{\mathrm{n}, \mathrm{u}} \mathrm{H}_{\mathrm{P}+4, \mathrm{Q}+3}^{\mathrm{M}+2, \mathrm{~N}+3}\left[\left.\mathrm{z}\{4(1+\mathrm{t})\}^{-\mathrm{T}}\right|_{\mathrm{B}} ^{\mathrm{A}}\right],
\end{aligned}
$$

where

$$
\begin{gathered}
A=(1-\mu, v),(1-\lambda-k j, \sigma),(1-\rho-\gamma \mathrm{u}, T),\left(a_{j}, \alpha_{j}\right)_{1, P^{\prime}}(1+a-b-\rho-\gamma u, T) \\
B=\left(\frac{1}{2}+\frac{a}{2}-\rho-\gamma u, T\right),\left(1+\frac{a}{2}-b-\rho-\gamma u, T\right),\left(b_{j}, \beta_{j}\right)_{1, Q^{\prime}}(1-\lambda-\mu-k j, \sigma+v) .
\end{gathered}
$$

The integral (6) is valid if the following sets of (sufficient) conditions are satisfied:

(i) $m$ is arbitrary positive integer, and the coefficient $A_{n, u}(n, u \geq 0)$ are arbitrary constants, real or complex.

(ii) $\Omega=\sum_{\mathrm{j}=1}^{\mathrm{N}} \alpha_{\mathrm{j}}-\sum_{\mathrm{j}=\mathrm{n}+1}^{\mathrm{P}} \alpha_{\mathrm{j}}+\sum_{\mathrm{j}=1}^{\mathrm{M}} \beta_{\mathrm{j}}-\sum_{\mathrm{j}=\mathrm{n}+1}^{\mathrm{Q}} \beta_{\mathrm{j}}>0$, and $|\arg \mathrm{z}|<(1 / 2) \Omega \pi$.

(iii) $\operatorname{Re}(1+\mathrm{a}-\mathrm{b})>0, \operatorname{Re}(\alpha)>-1, \operatorname{Re}(\beta)>-1, t>-1$,

$$
\gamma \geq 0, \operatorname{Re}(\lambda+\sigma \xi)>0, \operatorname{Re}(\rho+v \xi)>0,
$$

$\operatorname{Re}(\rho+\gamma \mathrm{u}+\mathrm{T} \xi)>0, \operatorname{Re}(1+\mathrm{a}-2 \rho-2 \gamma \mathrm{u}-2 \mathrm{~T} \xi)>0$

(iv) $\operatorname{Re}(1-2 b)>0$.

\section{References}

[1]. Gupta, Rajni: A Study of Generalized Fractional Operator and Multivariable Special Functions with Applications, Ph. D. thesis, Univ. of Rajsthan, India, 1988.

[2]. Rathie, N.: Integrals involving H-function, Vijnana Parishad Anusandhan Patrika, 22 (1979), 253 - 258.

[3]. Shrivastava, H. M.: A contour integral involving Fox's H-function, Indian J. Math. 14 (1972), 1 - 6.

[4]. Srivastava, H. S. P.: H-function of two variables I, Indore Univ., Res. J Sci. 5(1-2), p.87-93, (1978). 